\title{
Surface plasmon resonance of metal nanostructures as a complementary technique for microscopic size measurement
}

\author{
Ali Akbar Ashkarran ${ }^{*}$ and Alireza Bayat ${ }^{2}$
}

\begin{abstract}
Physical principals and the relations between Surface Plasmon Resonance (SPR) spectra and geometrical properties of metal nanostructures specially silver and gold were reported. Applying SPR technique enable us to investigate the accuracy of some simple equations for investigating geometrical properties of metal nanostructures based on their plasmonic spectrum. Geometrical properties, shape and size polydispersity of particles as well as particles' embedding medium refractive index are the crucial ignored parameters, effective in the accuracy of those equations. The restrictions of these relations in terms of shape, size distribution and refractive index of particles' embedding medium are also investigated and compared with other published reports. Furthermore, the equations of spherical particles have been extended to non-spherical ones and their accuracy has been investigated. It was found that the modified forms of the equations may lead to more exact results for non-spherical metal particles.
\end{abstract}

Keywords: Metal nanostructures; Surface plasmon resonance; Extinction spectra; Geometrical properties PACS: 52.80.Mg; 61.05.cp; 68.37.Lp; 81.16.Hc

\section{Background}

The two key parameters of nanostructures are their size and shape which have a crucial rule in their properties and diverse applications [1]. These key parameters are usually determined after synthesis of nanostructures using electron microscopic techniques such as transmission electron microscopy (TEM) and scanning electron microscopy (SEM). Although these techniques are very powerful in determination of size and shape of the nanostructures but the problem is that these techniques are not at hand anywhere and anytime. Moreover, microscopic techniques are too expensive compared with other measurement techniques such as optical spectroscopy. The optical properties of metallic nanostructures are determined by the collective oscillations of their conduction electrons with respect to the positive ion background, known as plasmon which relates the extinction coefficients to the size and geometrical properties of metallic nanostructures [2]. Therefore, optical spectroscopy is one of the simplest and easiest ways to determine

\footnotetext{
* Correspondence: ashkarran@gmail.com

'Department of Physics, Faculty of Basic Sciences, University of Mazandaran, Babolsar, Iran

Full list of author information is available at the end of the article
}

the geometrical properties of metal nanostructures including their size and shape [3]. The unique advantage of this property is the ability of determining the geometrical properties of nanostructures during the synthesis process which causes their controlled production.

The symmetry of nanostructures determines the ways that they are polarized, and the number of these ways correlates to the number of plasmonic peaks [4]. For example extinction spectra of small particles with the most symmetry shape i.e. sphere (Figure 1(a)) has just one peak because a small sphere could be polarized only in dipole mode, while cubic particles could be polarized both in dipole (in about $530 \mathrm{~nm}$ ) and quadrupole modes (in about $470 \mathrm{~nm}$ (Figure 1(b)). Therefore, they have two plasmonic peaks and the polarization modes are shown in Figure 2(a) and (b), respectively [5].

The symmetry also changes intensity of the extinction spectra. If surface charges are polarized according to mirror symmetry (without reflection plane which is bisector of corners) as depicted in Figure 2(c), the total dipole moment will increase, because accumulation of electrons in sharp corners are more than the other parts and in this polarization mode (Figure 2(d)) there will be four sharp corners instead of the two corners in Figure 2(c). 

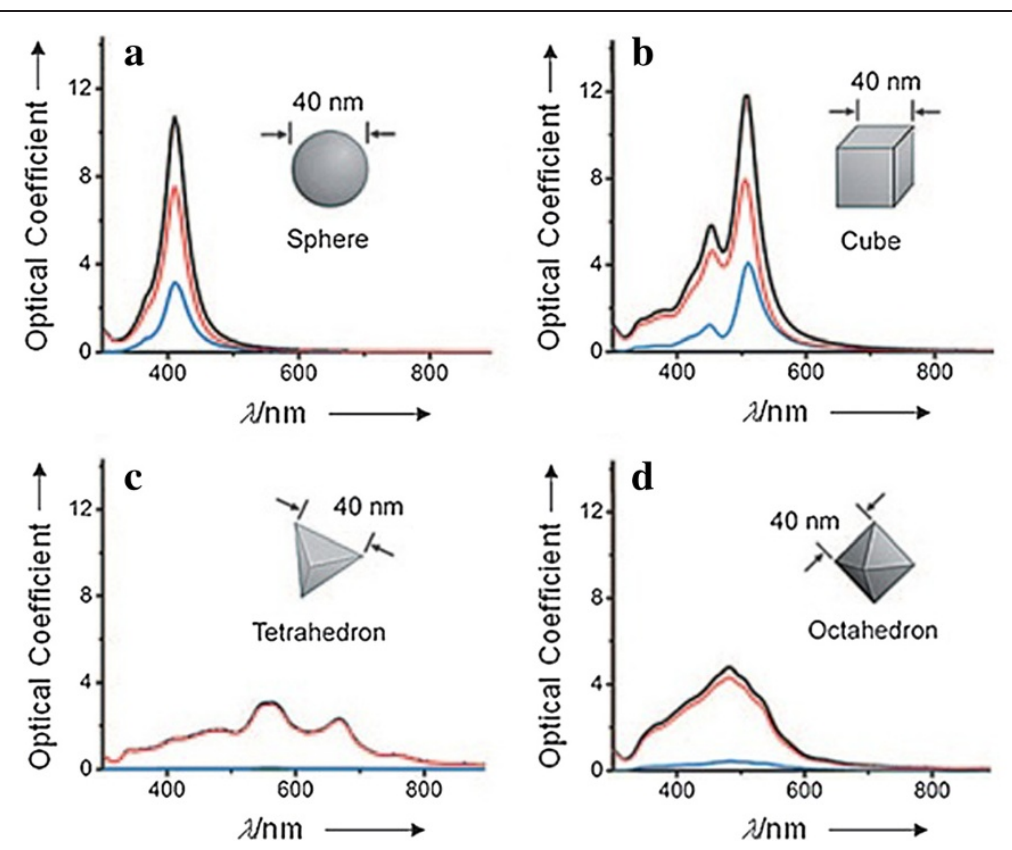

Figure 1 UV-Visible extinction (black), absorption (red), and scattering spectrum (blue) of Ag nanocrystals, illustrating the effect of shape on spectral characteristics: (a) sphere, (b) cube, (c) tetrahedron and (d) octahedron. Reprinted with permission from Xia et al. [4].
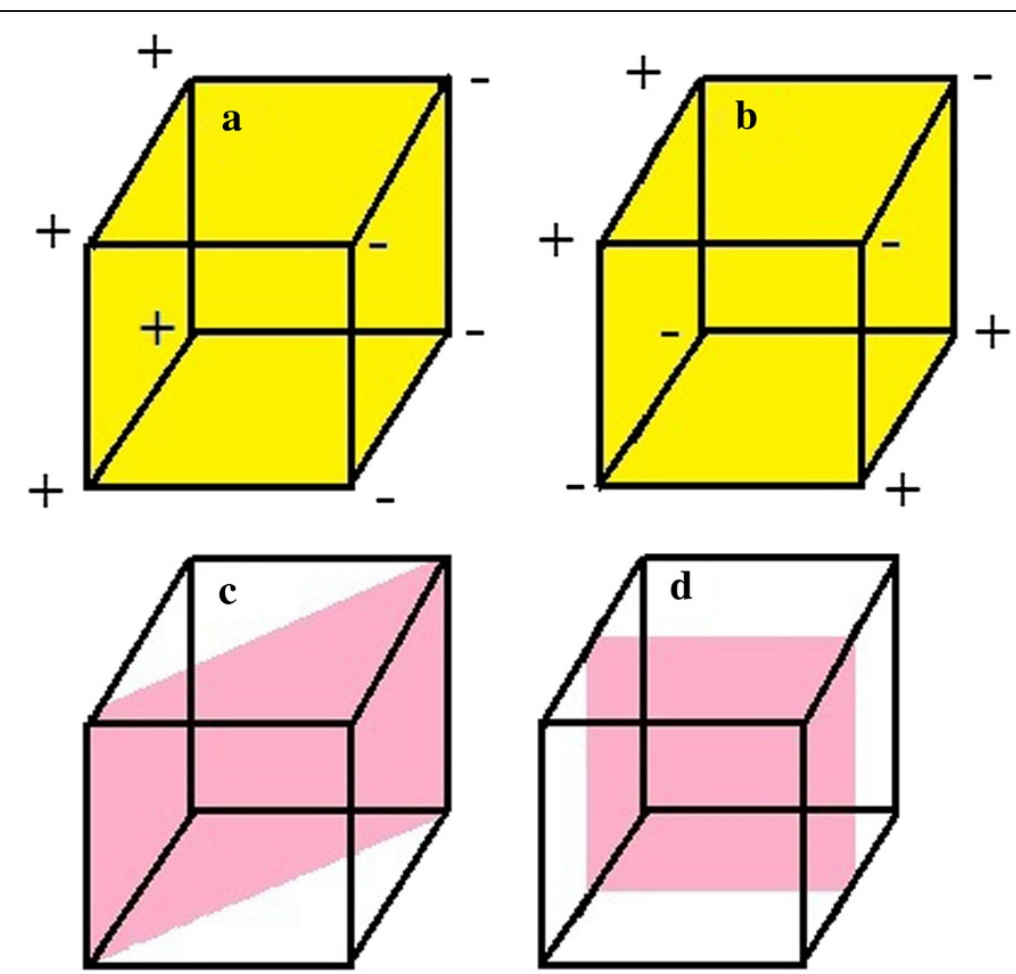

Figure 2 (a) Dipole, (b) quadrupole mode of a nanocube polarization, (c) and (d) the related symmetry planes of nanocubes in dipolar polarization. Reprinted with permission from Xia et al. and Zhang et al. [4,5]. 
Therefore, the number of plasmons will increase which increases the intensity of extinction spectra [4]. Another reason for decreasing the intensity of the extinction spectra is that electrons in silver nanocubes accumulate in corners (the corners are face to face), but in silver tetrahedrons, each corner is in front of a plane, so the strength of the effective dipole moment decreases and as a result the intensity of extinction spectra decreases (Figure 1(c)). Consequently, the extinction spectrum of octahedron is blue shifted (maybe due to more distance of face-to-face corners in octahedron) and is more intensive than tetrahedron (Figure 1(d)) [4].

The wavelength of plasmon resonance is affected by both particle size and refractive index of embedding medium [4,6-10]. In large particles surfaces are far enough from each other and electrons need less energy to shift from their equilibrium positions and consequently the effective restoring force applying to electrons by the ions decreases, compared with smaller particles. In contrast with nanocubes, one dimensional nanostructures have two dipole plasmonic peaks, one belongs to oscillating charges along longitudinal axis, and another belongs to oscillating charges along latitudinal axis [4]. Increasing the aspect ratio of these nanostructures not only causes a red shift in dipolar longitudinal plasmonic peak, but also increases intensity of the plasmonic peak.
Consequently, more plasmons will be excited and the intensity of their dipolar longitudinal plasmonic peaks will increase, too. This phenomena is illustrated both experimentally and theoretically (using discrete dipole approximation) for silver nanobars in Figure 3(a) and Figure 3(b), respectively.

As was mentioned earlier, sharp corners causes red shift in plasmonic peak due to accumulation of electrons in the corners and large distance among the electrons and positive ions. In very small nanostructures, extinction cross section is determined mainly by absorption cross section, in contrast with larger structures in which is determined mainly by scattering cross section, as is proofed by calculating absorption and scattering coefficients using Mie's theory [11]. Comparison of scattering coefficients in large enough nanobars and nanorices illustrates that sharp corners of nanostructures causes red shift of their extinction spectra (Figure 3(a) and (c)). The linear relation between aspect ratio of one dimensional nanostructures and their longitudinal dipolar plasmonic peak is illustrated both theoretically and experimentally in Figure 3(d).

In this work, only very useful relations for this purpose for metal nanostructures with different shapes have been collected, but also some of those relations from spherical to non-spherical particles are expanded using Coronado and Schatz's equation $[10,12-17]$.
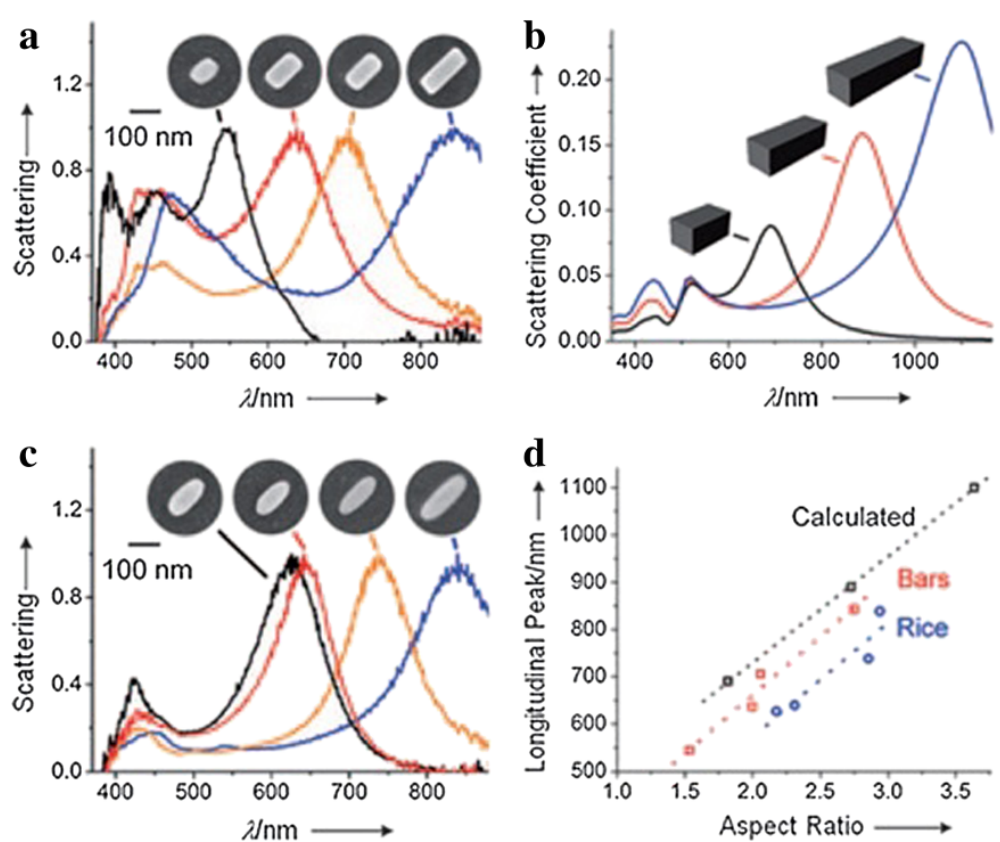

Figure 3 (a) SEM images of individual Ag nanobars and the corresponding normalized LSPR spectra. The longitudinal plasmon peak redshifts with increasing aspect ratio for the nanobars, (b) LSPR (scattering) spectra calculated using the DDA method for Ag nanobars (100, 150, and $200 \mathrm{~nm}$ in length, keeping width=55 nm and height=50 nm), (c) SEM images of individual nanorice with the corresponding normalized LSPR spectra and (d) plot of longitudinal plasmon peak location versus aspect ratio. The peaks of both nanobars and nanorice red-shift with increasing aspect ratio, but in average the peaks of nanobars are $80 \mathrm{~nm}$ red-shifted from nanorice. Reprinted with permission from Xia et al. [4]. 


\section{Methods}

In this section, simple empirical equations are presented which are used for determining geometrical properties of free-electron metal nanostructures. These relations are listed in Tables 1 and 2.

Khlebtsov derived an equation which relates average equivolume diameter $\left(\overline{\mathrm{d}_{\mathrm{ev}}}\right)$ of gold nanospheres to moderate inversion of spherical shape and their dipolar surface plasmon resonance wavelength $\left(\lambda_{\mathrm{SPR}}\right)$ using several experimental data, as following [16]:

$$
d_{\mathrm{ev}}=\left\{\begin{array}{c}
3+7.5 \times 10^{-5} \mathrm{X}^{4}, \mathrm{X}<23 \\
\frac{(\sqrt{\mathrm{X}-17}-1)}{0.06}, \mathrm{X} \geq 23
\end{array}, \mathrm{X}=\lambda_{\mathrm{SPR}}-500\right.
$$

He has also used linearity of $\frac{\mathrm{A}_{\max }}{\mathrm{A}_{\min }} \mathrm{V} . \mathrm{S} \cdot \ln \left(\overline{\mathrm{d}_{\mathrm{ev}}}\right)$ and $\mathrm{A}_{450}$ V.S. $\ln \left(\overline{\mathrm{d}_{\mathrm{ev}}}\right)$ graphs for determining $\overline{\mathrm{d}_{\mathrm{ev}}}$ nanoparticles with other properties (Table 1). In addition, Hoonacker and co-workers found that the graph of FWHM of extinction spectra of sliver nanospheres with smaller diameter than $20 \mathrm{~nm}$ versus the inverse of their diameter is linear and one may use this method for size determination of Ag NPs [14].

$e$ and $\sigma$ in Table 1 are the aspect ratio of spheroids and s-cylinders (cylinders with semi sphere ends) and standard normal distribution parameter that determines size distribution of nanoparticles, respectively. In a spheroid and s-cylinder $\overline{\mathrm{e}_{\mathrm{ev}}}$ may be calculated as:

$$
\overline{\mathrm{d}_{\mathrm{ev}}}=2 \mathrm{~b}\left[1+\frac{3\left(\mathrm{e}_{\mathrm{av}}-1\right)}{2}\right]^{1 / 3}
$$

Where

$$
\mathrm{e}_{\mathrm{av}}=\frac{\mathrm{a}_{\mathrm{av}}}{\mathrm{b}_{\mathrm{av}}}
$$

Also $\mathrm{A}_{\max }$ and $\mathrm{A}_{\min }$ in Table 1 are the maximum and minimum of extinction coefficient in extinction spectra.
Manikandan and co-workers [12,13] and Hoonacker et al. [14] have derived useful equations which relates diameter of nanospheres to their $\lambda_{\mathrm{SPR}}$ for all kind of metallic particles and silver particles respectively, as follows:

$$
\begin{aligned}
& \mathrm{R}=\frac{\mathrm{v}_{\mathrm{F}} \lambda_{\mathrm{SPR}}^{2}}{2 \pi \mathrm{c} \Delta \lambda} \beta \\
& \lambda_{\mathrm{SPR}}=382.6+1.18 \mathrm{~d}_{\mathrm{av}}
\end{aligned}
$$

Where $\Delta \lambda$ is full width at half maximum of extinction spectra (FWHM), $\mathrm{R}$ is particle radius, $\mathrm{C}$ is light velocity in vacuum $\left(\mathrm{c}=3 \times 10^{8} \mathrm{~m} / \mathrm{s}\right), \beta$ is proportionality constant, $v_{F}$ is Fermi velocity of electron gas in the particle which is equal to $1.4 \times 10^{6} \mathrm{~m} / \mathrm{s}$ for gold and silver particles and $d_{a v}$ in Eq. 5 is the average diameter of particles [18].

It should be mentioned that one may use Mie theory for simulation of extinction spectra of monodisperse metal and insulator spherical particles, and for extinction spectra of particles with non-spherical shapes T-Matrix simulation (linear transformation between coefficients of scattered and incident fields [19]) is the only exact solution [16,20-23].

Not only particle size, but also some other parameters possibly would be determined using surface plasmon resonance during production process. In quasi-static approximation, which the particles' dimensions are much less than incident wavelength, electromagnetic fields in the particle only depends on the time and standing plasmonic waves will be produced. In such a case the dependence of longitudinal resonance wavelength $\left(\lambda_{n}\right)$ to the resonance order ( $\mathrm{n}$ ) and aspect ratio (e) is given by this Equation [15]:

$$
\lambda_{\mathrm{n}} \simeq \mathrm{A}_{0}+\mathrm{A} \frac{\mathrm{e}}{\mathrm{n}}
$$

\begin{tabular}{|c|c|c|c|c|c|c|}
\hline Nanostructure shape & $\begin{array}{l}\text { Nanostructure } \\
\text { material }\end{array}$ & $\overline{\mathrm{dev}_{\mathrm{ev}}}$ limit $(\mathrm{nm})$ & $\sigma$ limit & e limit & Method & Ref. \\
\hline Spherical & Any material & Any size & $\sigma \approx 0$ & $\mathrm{e} \approx 0$ & Mie Theory & {$[16]$} \\
\hline $\begin{array}{l}\text { Spherical and quasi- } \\
\text { spherical }\end{array}$ & Any material & $1 \leq \overline{\mathrm{dev}_{\mathrm{ev}}} \leq 10$ & $\begin{array}{l}\text { Not } \\
\text { Determined }\end{array}$ & $1 \leq e \leq 1.2$ & $\begin{array}{l}\text { Using linear graph } \\
\frac{\mathrm{A}_{\max }}{\mathrm{A}_{\min }} \mathrm{V} \cdot \mathrm{S} \cdot \ln \left(\overline{\mathrm{d}_{\mathrm{ev}}}\right)\end{array}$ & [16] \\
\hline quasi-spherical & Any material & $3 \leq \overline{\mathrm{d}_{\mathrm{ev}}} \leq 70$ & $\begin{array}{l}\text { Not } \\
\text { Determined }\end{array}$ & $1.2 \leq e \leq 1.4$ & $\begin{array}{l}\text { Using linear graph } \\
\mathrm{A}_{450} \text { V.S. } \ln \left(\overline{\mathrm{d}_{\mathrm{ev}}}\right)\end{array}$ & [16] \\
\hline Spherical & Silver & Less than 20 & Not determined & Not determined & FWHM V.S. $\frac{1}{(\bar{d})}$ & [14] \\
\hline Spherical & Gold & $5 \leq \overline{\mathrm{dev}_{\mathrm{ev}}} \leq 100$ & Not determined & Moderate invert of sphere & Equation 1 & [16] \\
\hline Spherical & Silver & $5 \leq \overline{\mathrm{dev}_{\mathrm{ev}}} \leq 118$ & $\sigma<0.0001$ & Not determined & Equation 5 & [14] \\
\hline Spherical & Any material & Not determined & Not determined & Not determined & Equation 4 & {$[12,13]$} \\
\hline
\end{tabular}

Where $\mathrm{n}=1,2,3$, etc. corresponds to dipolar, quadrupolar, octapolar and etc. plasmon resonances.

Table 1 Different methods of determining $\overline{d_{e v}}$ for metal nanostructures 
Table 2 Methods of determining different geometrical parameters of nanostructures

\begin{tabular}{|c|c|c|c|c|c|c|c|}
\hline $\begin{array}{l}\text { Unknown } \\
\text { parameter }\end{array}$ & $\begin{array}{l}\text { Nanostructure } \\
\text { shape }\end{array}$ & $\begin{array}{l}\text { Nanostructure } \\
\text { material }\end{array}$ & Nanostructure size & $\sigma$ limit & e limit & Method & Ref. \\
\hline e (aspect ratio) & $\begin{array}{l}\text { Spheroid and } \\
\text { nanorod }\end{array}$ & Any material & Not determined & Not determined & Not determined & Equation 6 & [15] \\
\hline h (shell thickness) & spherical & Any material & $\begin{array}{l}\text { Quasi-Static approximation, } \\
\mathrm{h}_{\max } \approx \mathrm{d}_{\mathrm{ev}}\end{array}$ & Not determined & Not determined & Equation 8 & {$[10]$} \\
\hline$\Delta \mathrm{n}_{\mathrm{m}}{ }^{*}$ & Not determined & Any material & Not determined & Not determined & Not determined & Equation 7 & [15] \\
\hline
\end{tabular}

${ }^{*}$ Changes of embedding medium refraction index.

The constants $\mathrm{A}_{0}$ and $\mathrm{A}$ are independent of $\mathrm{n}$ factor which depend on the size and morphology of the particle. Surface plasmon resonances are very sensitive to changes of refractive index of their embedding medium upon thickness of their size [6-9]. Consequently this phenomenon makes free-electron metal nanoparticles excellent candidate for nanoscale sensors. Dependence of longitudinal dipolar and multipolar plasmon resonances to the changes of refractive index of embedding medium $\left(\Delta \mathrm{n}_{\mathrm{m}}\right)$ is given by [15]:

$$
\frac{\Delta \lambda_{\mathrm{SPR}}}{\lambda_{\mathrm{SPR}}} \simeq \mathrm{B} \frac{\Delta \mathrm{n}_{\mathrm{m}}}{\mathrm{n}_{\mathrm{m}}}
$$

Where $\mathrm{n}_{\mathrm{m}}$ initial medium refractive index and $\mathrm{B}$ is is a weak function of particle shape and order of multipole resonances which reduces by increase in the order.

Muskens and co-workers [10] derived a simple equation for measuring thickness of dielectric shell $(\mathrm{h})$ around metal core with weakly dispersed imaginary part of dielectric function in core-shell systems embedded in dielectric medium in a quasi-static regime, which for usual dielectric shells like water and glass is expressed as:

$$
1-\mathrm{a} \simeq \frac{\Delta \lambda_{\mathrm{SPR}}(\mathrm{h})}{\Delta \lambda_{\mathrm{SPR}}^{\max }}
$$

Where "a" is the core volume fraction:

$$
\mathrm{a}=\frac{\mathrm{D}^{3}}{(\mathrm{D}+\mathrm{h})^{3}}
$$

and

$$
\begin{aligned}
& \Delta \lambda_{\mathrm{SPR}}(\mathrm{h})=\lambda_{\mathrm{SPR}}(\mathrm{h})-\lambda_{\mathrm{SPR}}(\mathrm{h}=0) \\
& \Delta \lambda_{\mathrm{SPR}}^{\max }=\lambda_{\mathrm{SPR}}(\mathrm{h}=0)-\lambda_{\mathrm{SPR}}(\mathrm{h} \rightarrow \infty)
\end{aligned}
$$

$\lambda_{\mathrm{SPR}}(\mathrm{h}=0)$ and $\lambda_{\mathrm{SPR}}(\mathrm{h} \rightarrow \infty)$ define as surface plasmon resonance wavelength when there is not any shell around the core $(\mathrm{h}=0)$ and when the shell is too thick $(\mathrm{h} \rightarrow \infty)$ which the core does not sense embedding medium.

The parameters, which are describe by equations (6-11), and their applications are listed in Table 2. Tables 1 and 2 are very helpful for researches who are interested in controlling the production process of free-electron metal nanostructures.
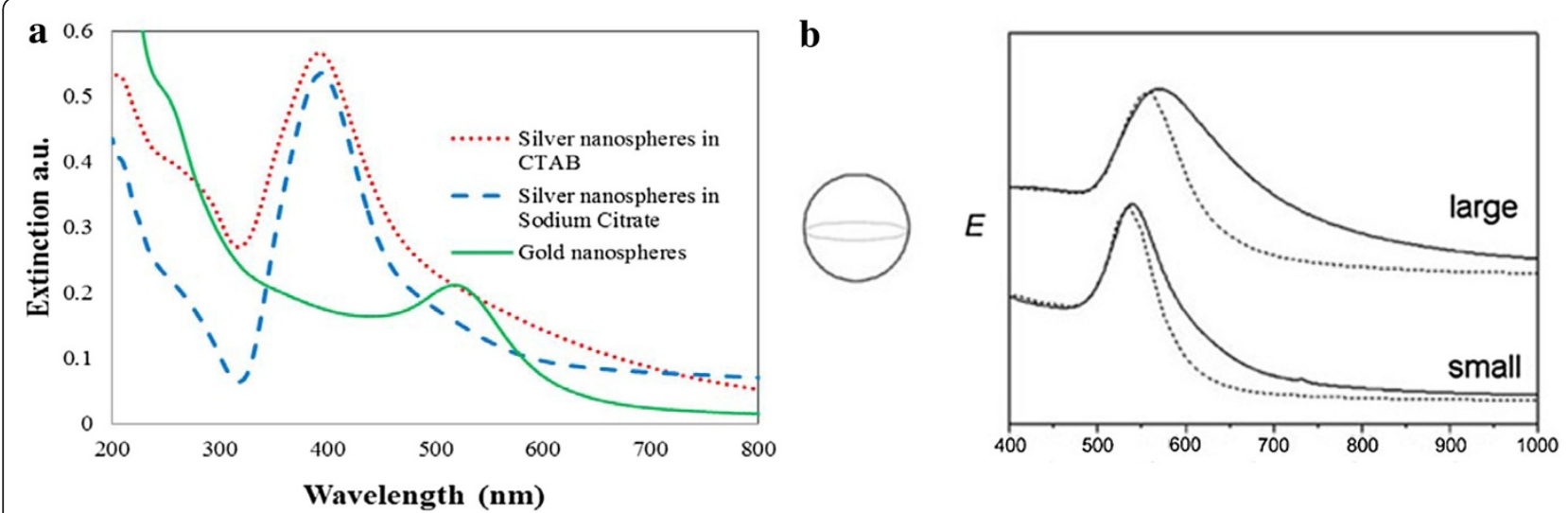

Figure 4 Extinction spectra of (a) gold and silver nanosphere produced by Ashkarran et al. [24,25,27] and (b) gold nanospheres produced by Seo et al. [26] (solid line shows experimental spectra and dotted lines show the result of DDA calculation). 
Table 3 Data of Figure 4 and calculated amounts of $\overline{d_{e v}}$ and $\beta$ for nanospheres

\begin{tabular}{|c|c|c|c|c|c|c|}
\hline Material & $\lambda_{\mathrm{SPR}}(\mathrm{nm})$ & FWHM (nm) & $\overline{\mathrm{dev}_{\mathrm{ev}}}(\mathrm{nm})$ & Calculated $\overline{\mathrm{d}_{\mathrm{ev}}}(\mathrm{nm})$ & $\beta$ & Ref. \\
\hline Silver in $C T A B$ & 393 & 142 & 14 & 8.81 & 8.66 & {$[25,27]$} \\
\hline Silver in sodium citrate & 394 & 101 & 30 & 9.66 & 13.14 & {$[25,27]$} \\
\hline Gold & 519 & 118 & 8 & 12.77 & 2.36 & {$[24,25]$} \\
\hline Gold & 542.1 & 126.3 & $49 \pm 5$ & 66.83 & 14.17 & [26] \\
\hline Gold (calculated by DDA) & 535 & 80.7 & 49 & 54.04 & 9.30 & [26] \\
\hline Gold & 573.7 & Incalculable & $81 \pm 8$ & 108.83 & Incalculable & {$[26]$} \\
\hline Gold (calculated by DDA) & 559.6 & Incalculable & 81 & 92.11 & Incalculable & {$[26]$} \\
\hline
\end{tabular}

In classical mechanics point of view, the mean free path (MFP) of electrons decreases by scattering from clusters' or particles' surface [11], Coronado and Schatz used a geometrical method to derive a practical equation for calculation of MFP in nanostructures [17]:

$$
\mathrm{L}_{\mathrm{eff}}=\frac{4 \mathrm{~V}}{\mathrm{~s}}
$$

This equation has been used to expand Equations $(1,4,5)$ to non-spherical shaped nanostructures to investigate the applicability of these equations about them.

$$
\begin{aligned}
& \mathrm{L}_{\text {eff }}=\left\{\begin{array}{r}
2+5 \times 10^{-5} \mathrm{X}^{4}, \mathrm{X}<23 \\
\frac{(\sqrt{\mathrm{X}-17}-1)}{0.09}, \mathrm{X} \geq 23
\end{array}, \mathrm{X}=\lambda_{\mathrm{SPR}}-500\right. \\
& \mathrm{L}_{\text {eff }}=\frac{2}{3} \frac{\mathrm{v}_{\mathrm{F}} \lambda_{\text {SPR }}^{2} \beta}{\pi \mathrm{r} \Delta \lambda} \\
& \lambda_{\mathrm{SPR}}=382.6+1.77 \mathrm{~L}_{\mathrm{eff}}
\end{aligned}
$$

Although, these equations have some strength points but they also have some weaknesses, which will be illustrated in the next section. In fact, some of those results possibly may not be exact due to the effects of shape, size distribution of particles and refractive index of embedding medium on extinction spectra, which are not considered in those equations.

\section{Result and discussion}

In this section, the applicability of Equations (1, 4, 5, 13-15) for gold and silver nanoparticles with different shapes is investigated. At first, the reliability of Equations $(1,4,5)$ about spherical particles is investigated and then the equations are expanded to particles with nonspherical shapes. Extinction spectra of spherical gold nanoparticles produced by Ashkarran and co-workers $[24,25]$ and Seo et al. [26] are shown in Figure 4. The data of these spectra, calculated values of $\overline{\mathrm{d}_{\mathrm{ev}}}$ and $\beta$ are shown in Table 3. Note that it was doubled the half of FWHM whenever it was impossible to be measured exactly.

It is expected that the plasmonic peak of silver nanoparticles in sodium citrate have more red shift than in CTAB because their average diameter is larger, but there is blue shift in their spectra compared with spectra of silver nanoparticles in CTAB. Because refractive index of CTAB (1.4 [28]) is larger than of sodium citrate (1.3 [29]), there is not any remarkable red shift in resonance wavelength of particles in sodium citrate. This is one of
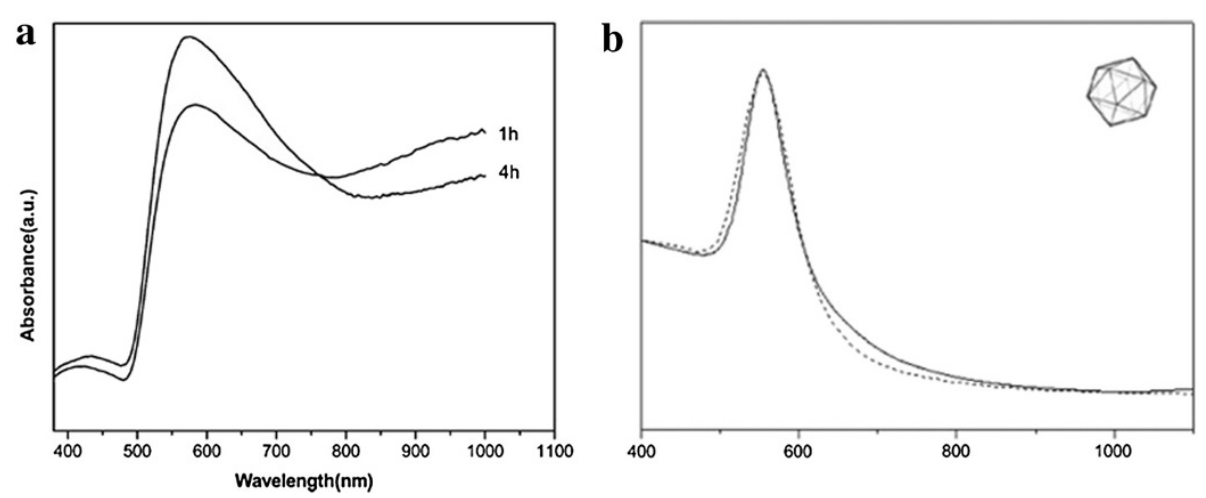

Figure 5 Extinction spectra of (a) Au icosahedra and their truncated forms [30] and (b) experimental and theoretical spectra of Au icosahedra. Reprinted with permission from Seo et al. [31]. 
Table 4 Calculated amounts of $\overline{d_{e v}}, L_{e f f}$ and $\beta$ for $A u$ icosahedra and their truncated forms

\begin{tabular}{lllllll}
\hline Shape & $\begin{array}{l}\text { Average } \\
\text { size }(\mathbf{n m})\end{array}$ & $\begin{array}{l}\boldsymbol{\lambda}_{\text {SPR }} \\
(\mathbf{n m})\end{array}$ & $\begin{array}{l}\text { FWHM } \\
(\mathbf{n m})\end{array}$ & $\begin{array}{l}\text { Calculated } \\
\text { size }(\mathbf{n m})\end{array}$ & $\boldsymbol{\beta}$ & Ref. \\
\hline $\begin{array}{l}\text { Truncated } \\
\text { icosahedral }\end{array}$ & 222.48 & 574.7 & 120.3 & 109.93 & 54.55 & {$[30]$} \\
$\begin{array}{l}\text { icosahedral } \\
\begin{array}{l}\text { Icosahedral } \\
\text { (experimental) }\end{array}\end{array}$ & 253.45 & 583 & 135 & 118.73 & 67.77 & {$[30]$} \\
$\begin{array}{l}\text { Icosahedral } \\
\text { (teoritical) }\end{array}$ & 94 & 561.96 & 101.96 & 95.08 & 20.43 & {$[31]$} \\
\hline
\end{tabular}

the weaknesses of Eq. 5 which in spite of high sensitivity of silver nanoparticles to the changes of refractive index of their embedding medium, the equation does not have any dependence to this important parameter. It seems that the difference between calculated $\overline{\mathrm{d}_{\mathrm{ev}}}$ for particles in $\mathrm{CTAB}$ and the measured one is mainly due to this weakness. Another weakness is that Hoonacker and coworkers did not considered the required limits of $\sigma$ in their equation.

Similarly, Eq. 4 does not depend on refractive index of particles' embedding medium nor size distribution. It was expected that $\beta$ values to be same for different silver samples but they were not, due to different values of these parameters.

Equation 1, which was obtained by Khlebtsovand coworkers, does not depend on refractive index of embedding media, shape and size distribution of particles, too. So, in spite of approximate results, for exact results the constants in this equation should be replace with functions of above parameters. Considering these points in Equations 4 and 5 the accuracy and generality of these equations would increase. Note that particles with same shape have the approximately same $\beta$ values.

Now the validity of Equations 1 and 4 for truncated icosahedrons [30] and icosahedrons [30,31] gold nanoparticles are investigated. The extinction spectra of these structures are shown in Figure 5. In Table 4, experimental and calculated values of $\bar{d}$ and $\beta$ are presented. The average size of particles has been considered as their equivolume diameter for samples of Ref. [30] and [31].

Equation 1 leads to exact results for both theoretical and experimental data of Ref. [31]. So, one could expect validity of this equation for dipolar resonance of other regular polyhedral nanoparticles. Note that the results of gold nanospheres in Table 1 were not exact probably because the effect of refractive index of embedding

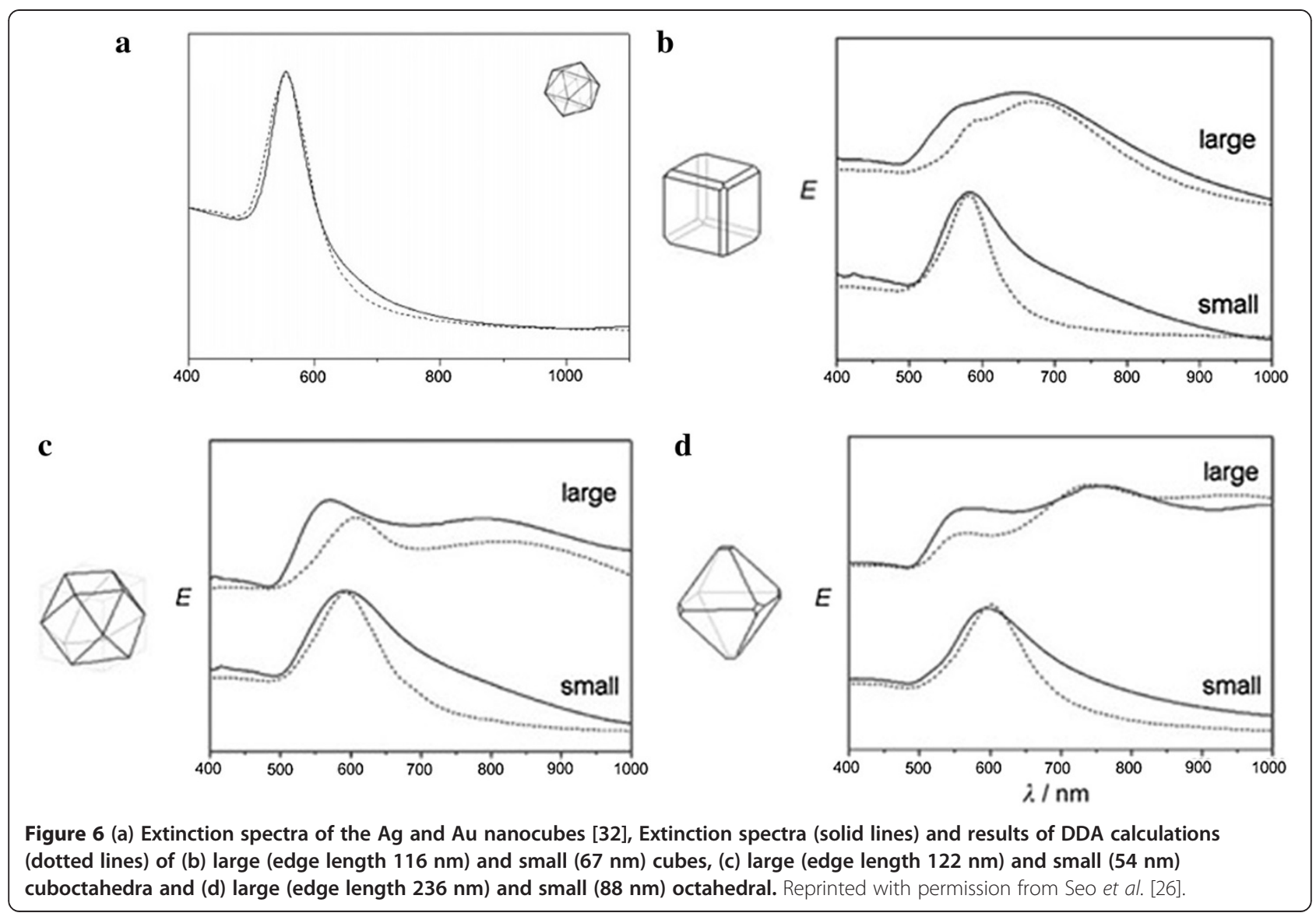


Table 5 Calculated values of edge size, $\overline{d_{e v}}, L_{\text {eff }}$ and $\beta$ for Au nanoparticles with different shapes

\begin{tabular}{|c|c|c|c|c|c|c|c|c|c|c|c|c|c|c|}
\hline Ref. & [26] & [26] & [26] & [26] & [32] & [32] & [26] & [26] & [26] & [26] & [26] & [26] & [26] & [26] \\
\hline $\begin{array}{l}\beta \text { using } \\
L_{\text {eff }}\end{array}$ & 22.89 & 38.16 & Incalculable & Incalculable & 17.26 & 60.28 & 14.31 & 26.35 & Incalculable & Incalculable & 20.26 & 32.77 & Incalculable & Incalculable \\
\hline$\frac{\beta \text { using }}{{\overline{d_{\mathrm{ev}}}}}$ & 25.29 & 42.17 & Incalculable & Incalculable & 21.42 & 74.79 & 17.75 & 32.69 & Incalculable & Incalculable & 23.96 & 38.75 & Incalculable & Incalculable \\
\hline $\begin{array}{l}\text { Calculated } \\
\mathrm{L}_{\text {eff }}(\mathrm{nm})\end{array}$ & 84.59 & 84.59 & 182.61 & 172.87 & 64.24 & 85.53 & 77.56 & 78.74 & 129.52 & 118.27 & 90.11 & 84.59 & 218.91 & 233.08 \\
\hline $\begin{array}{l}\text { Calculated } \\
\overline{\mathrm{d}_{\mathrm{ev}}}(\mathrm{nm})\end{array}$ & 126.89 & 126.89 & 273.92 & 259.31 & 96.37 & 128.30 & 116.35 & 118.11 & 194.28 & 177.41 & 135.17 & 126.89 & 328.37 & 349.62 \\
\hline $\begin{array}{l}\text { Calculated } \\
\text { edge (nm) }\end{array}$ & 76.84 & 76.84 & 165.89 & 157.04 & 77.67 & 103.41 & 93.77 & 95.19 & 156.58 & 142.99 & 139.98 & 131.41 & 340.06 & 362.07 \\
\hline $\begin{array}{l}\text { FWHM } \\
(\mathrm{nm})\end{array}$ & 147.3 & 245.6 & Incalculable & Incalculable & 105.6 & 304 & 107 & 198.2 & Incalculable & Incalculable & 150.8 & 236.8 & Incalculable & Incalculable \\
\hline$\lambda_{\text {SPR }}(\mathrm{nm})$ & 591.2 & 591.2 & 821.0 & 791.2 & 563 & 534 & 580.7 & 582.4 & 677.2 & 652.6 & 600 & 591.2 & 945.6 & 1000 \\
\hline Kind & Theoretical & Experimental & Theoretical & Experimental & Experimental & Experimental & Theoretical & Experimental & Theoretical & Experimental & Theoretical & Experimental & Theoretical & Experimental \\
\hline $\mathrm{L}_{\text {eff }}(\mathrm{nm})$ & 53.79 & 53.79 & 121.53 & 121.53 & 51.33 & 56 & 44.66 & 44.66 & 77.33 & 77.33 & 47.90 & 47.90 & 128.46 & 128.46 \\
\hline$\overline{\mathrm{d}_{\mathrm{ev}}}(\mathrm{nm})$ & 89.16 & 89.16 & 201.44 & 201.44 & 95.53 & 104.21 & 83.126 & 83.126 & 143.92 & 143.92 & 84.97 & 84.97 & 227.88 & 227.88 \\
\hline Edge (nm) & 54 & $54 \pm 8$ & 122 & $122 \pm 13$ & $77 \pm 6$ & $84 \pm 6$ & 67 & $67 \pm 6$ & 116 & $116 \pm 11$ & 88 & $88 \pm 10$ & 236 & $236 \pm 19$ \\
\hline Shape & Cuboctagonal & Cuboctagonal & Cuboctagonal & Cuboctagonal & Cubic & Cubic & Cubic & Cubic & Cubic & Cubic & Octahedral & Octahedral & Octahedral & Octahedral \\
\hline Material & Gold & Gold & Gold & Gold & Gold & Silver & Gold & Gold & Gold & Gold & Gold & Gold & Gold & Gold \\
\hline
\end{tabular}


medium is not considered in Eq. 1. Different values obtained for $\beta$ considering the data of Ref. [31] show the effect of size distribution in validity of Eq. 4. Also different calculated $\beta$ values for Ref. [30] and [31] are probably due to effect of refractive index on this equation, since small changes in referactive index lead to changes in $\lambda_{\text {SPR }}$. It is important to note that embedding mediums of nanoparticles of Ref. [30] are the same and the large difference between calculated $\beta$ values is propably due to shape effects of NPs on their extinction spectra. So, for overgeneralizing Eq. 4 one should considers shape and size distribution effects, as well as the effects of refractive index of embedding medium on extinction spectra.

Now the validity of Equations 1, 4, 5, 13, 14 and 15 about cuboctahedral, octahedral and cubic gold and silver nanoparticles are investigatedusing data in Ref. [26] and [32]. Their extinction spectra are depicted in Figure 6 (theoreticalspectra obtained using DDA calculation), and the calculated values of $\overline{\mathrm{d}_{\mathrm{ev}}}, \mathrm{L}_{\mathrm{eff}}$, and $\beta$ using both $\overline{\mathrm{d}_{\mathrm{ev}}}$ and $\mathrm{L}_{\mathrm{eff}}$ are listed in Table 5 .

Differences between theoretical and experimental values of $\beta$ for cuboctahedra, cubes and octahedral may due to size distribution and in this regard dispersity should be considered in Eq. 4 and 14. Although refractive index of the embedding medium of the cuboctahedral particles affects the final results but, for larger particles Eq. 1 and 13 are not applicable since their equivolumediameters are larger than that Khlebtsov determined.

Exact calculated value of edge size of cubic gold particles prepared by Lee and co-workers [32] show the applicability of Eq. 1 and 13 for non-spherical particles if the effects of shape, size distribution and medium were considered [32]. Moreover, the results obtained for silver nanostructures confirm this expression and reveal that $\beta$ depends on particles' matter. It seems that for particles with sharp corners like octahedra, these equations are not applicable. Because sharp corners affect extinction spectra and cause such a high red shift in extinction spectra which modifications of Eq. 1 and 4 would not lead to exact results. Therefore, Eq. 13, 14 and 15 are more exact for non-spherical particles since they deal with MFP of electrons which is determining in electrical and optical properties of nanoparticles, independent of their shapes.

\section{Conclusion}

The applicability of size determination equations based on extinction spectra of spherical and non-spherical silver and gold nanoparticles have been invistigated. It has been found that modified forms of those equations may give more exact results and they should be modified as a function of shape, size distribution and refractive index of particles' embedding medium in order to be overgeneralized to non-spherical particles instead of sphere only. Furthermore, equations of spherical particles should depend on these parameters to rise to more exact results. Moreover, it has been found that modified forms of those equations do not lead to exact results for particles which have corners with spatial angels less than $\pi / 2$ sr such as octahedral particles. Such sharp corners cause additional redshift in the extinction spectra which has not been considered in those equations.

\begin{abstract}
Abbreviations
SPR: Surface Plasmon Resonance; $\overline{d_{\mathrm{ev}}}$ : Average equivolume diameter; $\lambda_{\text {sPR: }}$ Surface plasmon resonance wavelength; $e$ : The aspect ratio of spheroids and $\mathrm{s}$-cylinders; $\sigma:$ Standard normal distribution parameter; $A_{\text {max }}$ : Maximum of extinction coefficient in extinction spectra; $A_{\text {min }}$ : Minimum of extinction coefficient in extinction spectra; $\mathrm{A}_{450}$ : Extinction coefficient in $450 \mathrm{~nm}$ in extinction spectra; $\mathrm{a}_{\mathrm{av}}$ : The average major semi-axis; $\mathrm{b}_{\mathrm{av}}$ : The average minor semi-axis; $\Delta \lambda$ : Full width at half maximum of extinction spectra (FWHM); R: Particle radius; $C$ : Light velocity in vacuum; $\beta$ : Proportionality constant; $v_{F}$ : Fermi velocity of electron gas in the particle; $d_{a v}$ : The average diameter of particles; $\lambda_{n}$ : Longitudinal resonance wavelength where $n=1,2$, 3, etc. corresponds to dipolar, quadrupolar, octapolar and etc. plasmon resonances; $\mathrm{A}_{0}$ : Constant; $\mathrm{A}$ : Constant; $\mathrm{n}_{\mathrm{m}}$ : Initial medium refractive index; B: A weak function of particle shape and order of multipole resonances; $\Delta n_{m}$ : Changes of refractive index of the embedding medium; $h$ : Thickness of dielectric shell around metal core with diameter D; a: The core volume fraction; $L_{\text {eff: }}$ The mean free path in nanostructures; $V$ : The volume of particles; S: The surface area of particles.
\end{abstract}

\section{Competing interests}

The authors declare that they have no competing interests.

\section{Authors' contributions}

AAA carried out the absorption spectroscopy studies, participated in the derivation of equations and prepared the manuscript. $A B$ carried out the derivation of equations and drafted the manuscript. All authors read and approved the final manuscript.

\section{Acknowledgements}

This work was supported by the Iran national science foundation (INSF). Authors express their special thank to Mrs Effat Zandevakili for great helpful discusions.

\section{Author details}

${ }^{1}$ Department of Physics, Faculty of Basic Sciences, University of Mazandaran, Babolsar, Iran. ${ }^{2}$ Plasma Physics Research Center, Science and Research Branch, Islamic Azad University, Tehran, Iran.

Received: 29 May 2013 Accepted: 17 August 2013

Published: 30 Aug 2013

\section{References}

1. Wiley, B, Sun, Y, Mayers, B, Xia, Y: Shape-Controlled Synthesis of Metal Nanostructures: The Case of Silver. CHEM-EUR J11, 454-463 (2005)

2. Kittle, C: Introduction to Solid State Physics. 5th edition, Wiley Eastern Limited (1983)

3. Rycenga, M, Cobley, CM, Zeng, J, Li, W, Moran, CH, Zhang, Q, Qin, D, Xia, Y: Controlling the Synthesis and Assembly of Silver Nanostructures for Plasmonic Applications. Chem Rev 111, 3669-3712 (2011)

4. Xia, Y, Xiong, Y, Lim, B, Skrabalak, SE: Shape-Controlled Synthesis of Metal Nanocrystals: Simple Chemistry Meets Complex Physics? AngewChemInt 48, 60--103 (2009)

5. Zhang, S, Bao, K, Halas, NJ, Xu, H, Nordlander, P: Substrate-Induced Fano Resonances of a PlasmonicNanocube: A Route to Increased-Sensitivity Localized Surface Plasmon Resonance Sensors Revealed. Nano Lett 11, 1657-1663 (2011)

6. Willets, KA, Van Duyne, RP: Localized Surface Plasmon Resonance Spectroscopy and Sensing. Annu Rev PhysChem 58, 267-297 (2007) 
7. Alekseeva, AV, Bogatyrev, VA, Khlebtsov, BN, Mel'nikov, AG, Dykman, LA, Khlebtsov, NG: Gold nanorods: Synthesis and optical properties. Colloid 68, 661-678 (2006)

8. Khlebtsov, NG, Trachuk, LA, Melnikov, AG: Plasmon resonances of silver and gold nanorods. Proc SPIE 5475, 1-11 (2004)

9. Khlebtsov, NG, Trachuk, LA, Mel'nikov, AG: The effect of the size, shape, and structure of metal nanoparticles on the dependence of their optical properties on the refractive index of a disperse medium. Opt Spectrosc 98, 77-83 (2005)

10. Muskens, OL, Billaud, P, Broyer, M, Del Fatti, N, Vallée, F: Optical extinction spectrum of a single metal nanoparticle: Quantitative characterization of a particle and of its local environment. Phys. Rev. B 78, 205410-205418 (2008)

11. Kreibig, U, Vollmer, M: Optical Properties of Metal Clusters. Springer, Berlin, Germany (1995)

12. Picciotto, A, Torrisi, L, Margarone, D, Bellutti, P: Particle size determination of silver nanoparticles generated by plasma laser ablation using a deconvolution method. RADIAT EFF DEFECT S 165, 706-712 (2010)

13. Manikandan, D, Mohan, S, Magudapathy, P, Nair, KGM: Blue shift of plasmon resonance in $\mathrm{Cu}$ and $\mathrm{Ag}$ ion-exchanged and annealed soda-lime glass: an optical absorption study. Physica B 325, 86-91 (2003)

14. Hoonacker, AV, Englebienne, P: Revisiting Silver Nanoparticle Chemical Synthesis and Stability by Optical Spectroscopy. CurrNanosci 2, 359-371 (2006)

15. Khlebtsov, BN, Khlebtsov, NG: MultipolePlasmons in Metal Nanorods: Scaling Properties and Dependence on Particle Size, Shape, Orientation, and Dielectric Environment. J PhysChem C111, 11516-11527 (2007)

16. Khlebtsov, NG: Determination of Size and Concentration of Gold Nanoparticles from Extinction Spectra. Anal Chem 80, 6620-6625 (2008)

17. Coronado, EA, Schatz, GC: Surface plasmon broadening for arbitrary shape nanoparticles: A geometrical probability approach. J ChemPhys 119, 3926-3934 (2003)

18. Ziman, JM: Principles of the Theory of Solids, p. 278. Cambridge University Press, Cambridge, U.K (1979)

19. Bohren, CF: Huffman. Absorption and Scattering of Light by Small Particles John Wiley \& Sons, D.R. (1983)

20. Khlebtsov, NG, Dykman, LA: Optical properties and biomedical applications of plasmonic nanoparticles. J Quant SpectroscRadiat Transfer $111,1-35(2010)$

21. Debye, P: Der Lichtdruck auf Kugeln von beliebigem Material. Ann Phys 335, 57-136 (1909)

22. Scaffardi, LB, Pellegri, N, Sanctis, OD, Tocho, JO: Sizing gold nanoparticles by optical extinction spectroscopy. Nanotechnology 16, 158-163 (2005)

23. Alekseeva, AV, Bogatyrev, VA, Dykman, LA, Khlebtsov, BN, Trachuk, LA, Melnikov, AG, Khlebtsov, NG: Preparation and optical scattering characterization of gold nanorods and their application to a dotimmunogold assay. Appl Opt 44, 6285-6295 (2005)

24. Ashkarran, AA, Irajizad, A, Mahdavi, SM, Ahadian, MM, HormoziNezhad, MR: Rapid and efficient synthesis of colloidal gold nanoparticles by arc discharge method. ApplPhys A96, 423-428 (2009)

25. Ashkarran, AA: Metal and Metal Oxide Nanostructures Prepared by Electrical Arc Discharge Method in Liquids. J Cluster Sci 22, 233-266 (2011)

26. Seo, D, Yoo, Cl, Park, JC, Park, SM, Ryu, S, Song, H: Directed Surface Overgrowth and Morphology Control of Polyhedral Gold Nanocrystals. AngewChemlnt 47, 763-767 (2008)

27. Ashkarran, AA, Irajizad, A, Ahadian, MM, HormoziNezhad, MR: Stability, size and optical properties of colloidal silver nanoparticles prepared by electrical arc discharge in water. EurPhys J ApplPhys 48, 10601-10607 (2009)

28. Yu, C, Varghese, L, Irudayaraj, J: Surface Modification of Cetyltrimethylammonium Bromide-Capped Gold Nanorods to Make Molecular Probes. Langmuir 23, 9114-9119 (2007)

29. Salabat, A, Shamshiri, L, Sahrakar, F: Thermodynamic and transport properties of aqueous trisodium citrate system at $298.15 \mathrm{~K}$. J MolLiq $118,67-70(2005)$

30. Xu, J, Li, S, Weng, J, Wang, X, Zhou, Z, Yang, K, Liu, M, Chen, X, Cui, Q, Cao, M, Zhang, Q: Hydrothermal Syntheses of Gold Nanocrystals: From Icosahedral to Its Truncated Form. AdvFunct Mater 18, 277-284 (2008)
31. Seo, D, Yoo, Cl, Chung, IS, Park, SM, Ryu, S, Song, H: Shape Adjustment between Multiply Twinned and Single-Crystalline Polyhedral Gold Nanocrystals: Decahedra, Icosahedra, and Truncated Tetrahedra. J PhysChem $112,2469-2475$ (2008)

32. Lee, YH, Chen, $H, X u, Q$, Wang, J: Refractive Index Sensitivities of Noble Metal Nanocrystals: The Effects of Multipolar Plasmon Resonances and the Metal Type. J PhysChem C115, 7997-8004 (2011)

\subsection{6/2228-5326-3-50}

Cite this article as: Ashkarran and Bayat: Surface plasmon resonance of metal nanostructures as a complementary technique for microscopic size measurement. International Nano Letters 2013, 3:50

\section{Submit your manuscript to a SpringerOpen ${ }^{\odot}$ journal and benefit from:}

- Convenient online submission

- Rigorous peer review

- Immediate publication on acceptance

- Open access: articles freely available online

- High visibility within the field

- Retaining the copyright to your article

Submit your next manuscript at $>$ springeropen.com 